\title{
Proceedings of Seminar \#99: Surveillance Methodology, 1987 Annual Meeting, American Society for Microbiology
}

\author{
David Birnbaum, MPH; James M. Hughes, MD
}

Perfection of means and confusion of goals seem, in my opinion, to characterize our age.

\section{Albert Einstein Out of My Later Years}

Our understanding of nosocomial infection control has progressed from an era when largely untested extensions of logic provided very precise and specific rules of conduct for hospital personnel. Today, we can be much less confident of the value of many of these restrictive measures. We now better appreciate the complexities and subtleties involved in quantifying risks, in understanding modes of disease transmission, and in evaluating effectiveness of control measures. In this area of concern with humane yet cost-effective care, increasingly complex technologies, and greater appreciation of previously unrecognized but important reservoirs for disease transmission, the need for valid evaluation of risks and benefits is more critical than ever, presenting an important challenge and opportunity to hospital epidemiologists.

If we are to continue expanding our knowledge through applied research and communicating the findings in ways persuasive enough to achieve the potential benefits from that work, we must periodically re-examine our infection surveillance and control program strategies. Over the years, we have accumulated a wealth of guide-

From Victoria General Hospital, Victoria, British Columbia, Canada (Mr. Bimbaum) and the Hospital Infections Program, Center for Infectious Diseases, Centers for Disease Control, Public Health Service, US Department of Health and Human Services, Allanta, Georgia, (Dr. Hughes).

Mr. Birnbaum and Dr. Hughes were co-convenors at the 1987 Annual Meeting of the American Society for Microbiology, Atlanta, Georgia, March 3, 1987.

Address reprint requests to David Bimbaum, MPH, Victoria General Hospital, 35 Helmcken Road, Victoria, British Columbia V8Z 6R5, Canada. lines, standards, traditions, and published recommendations. We must periodically take stock of goals and achievements and reconsider the current validity of historic strategies, recognizing that individual hospitals may have different priorities, different problems, and different infection control program objectives.

Surveillance provides the cornerstone for our research apparatus, yet has remained a topic of controversy for many years. A slowly increasing number of publications offer insight into the relative merits of different approaches to infection control, data collection, data analysis, and use of information. In reviewing these alternatives, the proceedings of this seminar include perspectives of epidemiologists from diverse backgrounds working in a variety of environments, ranging from federal government agency to university teaching hospital to community hospital.

Nothing is ever done until everyone is convinced that it ought to be done, and has been convinced for so long, that it is now time to do something else . . . !

Frank Cornford

Although some sources have advised simple means to compute a hospital's "infection rate," a concluding summary in Proceedings of the Second International Conference on Nosocomial Infections held in 1980 notes that the "best methods" for surveillance have not been identified. Surveillance methodology is not addressed in the Centers for Disease Control's Guidelines for the Prevention and Control of Nosocomial Infection series. The options are numerous and the fundamental issues complex.

Miettinen comments in Theoretical Epidemiology ${ }^{1}$ that epidemiology is more a philosophy and set of analytical techniques than a science per se. It is an investigative approach more so than a specific body of scientific knowledge, requiring skill, judgment and experience. In this 
vein, the proceedings of the seminar offer practitioners a summary of evolving methodologic issues, and administrators pragmatic criteria by which to judge program priorities. We do not offer a single approach for everyone, nor seek uniformity; the message is just the opposite.

Far better an approximate answer to the right question, which is of ten vague, than an exact answer to the wrong question, which can always be made more precise.

John Tukey, The Future of Data Analysis, Ann Math Statist 1962;33

The first presentations discuss surveillance objectives in a historical context. They outline evolving sophistication in descriptive and analytic epidemiologic tasks crucial to expanding our understanding of hospital-acquired infections. This topic leads to a consideration of intervention strategies and a discussion of approaches to minimizing the risk of both epidemic and endemic infections. Whether the intent of hospital epidemiologists or other infection control practitioners is to pursue similar lines of investigation or to adopt pragmatic "rules of thumb" gleaned from existing studies, these presentations should provide a useful guide to current information.

The final three presentations turn to questions of surveillance methodology: how can the objectives previously outlined be achieved? Advantages, disadvantages, and potential pitfalls are discussed in regard to using alternative data sources, different approaches to data analysis, or selecting between manual and automated systems. Established techniques to detect confounding or effect modification are illustrated and proposed techniques to select optimal outbreak thresholds are reviewed.

\section{REFERENCES}

1. Mietuinen O: Theoretical Epidemiology: Principle of Occumence Rescarch in Medicine. New York, John Wiley \& Sons, 1985. 